\title{
Identification of Multidrug-Resistant Bacteria in Intensive Care Unit Patients
}

\section{Rawan Sultan Alosaimi ${ }^{1}$, Nidaa M ahmoud Johari ${ }^{1}$, Sara M ahbob Alotaibi ${ }^{1}$, Bashyer Mater Albeladi ${ }^{1}$, Wafaa Ahmed Hamdi $^{1}$, Hayat Mathkor ${ }^{1}$, Khadiga Ahmed Ismail ${ }^{1 *}$, Laila Alotaibi ${ }^{2}$, Doaa Mahmoud Johari ${ }^{2}$, Manhal Shbat ${ }^{2}$, Khadija $^{2}$ Hassan Khan ${ }^{2}$}

${ }^{1}$ Taif University, Taif, Saudi Arabia

${ }^{2}$ King Faisal Medical Complex,Taif, Saudi Arabia

*Corresponding Author: Khadiga Ahmed Ismail, Taif University, Taif, Saudi Arabia.

Received: October 17, 2019; Published: November 11, 2019

DOI: $10.31080 /$ ASMI.2019.02.0431

\begin{abstract}
Background: Hospital-acquired infection (HAIs)is a crucial issue specially in ICU efforts have been done to prevent their occurrence and improve patient safety.

Objective: To identify hospital acquired microorganisms and the their antibiotic sensitivity in ICU patients and identify the percentage of multidrug-resistant bacteria (MDR).

Method: A 6-month retrospective cohort study was conducted to identify microorganisms caused HAI in patients in the intensive care unit (ICU) of King Faisl medical complex, Taif, KSA during the period from May 2018 to October 2018.

Results: During the study period, 1101 patients were admitted to ICU 137 patients develop infection only 15 (10.6\%) of them proved to be HAIs. The most frequent organisms were as follows: 31(23\%) Klebsiella Spp, 12\% of these were ESBL producers, 14 (10.2\%) $E$. coli,3.6\% of these were ESBL producers, 14 (10.2\%) Acinetobacter baumannii, all were MDR. and 12(9\%) Staph aureus, 1\% of these were MRSA.

Conclusions: The emergence of MDR bacteria poses a difficult task for physicians, who have limited therapeutic options. And therefore, a continuous surveillance program to observe the emergence of different bacterial resistance patterns is advised to establish unified guidelines across Saudi Arabia to reduce further progress in the emergence of MDR microorganisms.

Keywords: Hais; ICU; Nosocomial Infections; MDR; MRSA; ESBL
\end{abstract}

\section{Introduction}

According to the World Health Organization a Hospital Acquired Infection is, "an infection acquired in hospital by a patient who was admitted for a reason other than that infection. Also it is known as nosocomial infections which are those infections acquired in hospital or healthcare service unit that first appear 48 hours or more after hospital admission or within 3 days after discharge following in-patient care. Hospital acquired infections are a worldwide phenomenon. Patient care is provided in settings ranging from small health care clinics with basic facilities to large sophisticated highly equipped hospitals with state of the art technology. Despite progress in public health and hospital care, infections continue to develop in hospitalized patients and also in hospital staff. The World Health Organization (WHO) called HAIs a major cause of death and disability for patients [1].

Nosocomial infections are also one of the leading causes of death. Microorganisms are transmitted in hospitals by several routes and same microorganisms may be transmitted by more than one route. The main routes of transmission include contact, airborne, common vehicle and vector borne [2]

A survey on HAIs reveals that at any time, over 1.4 million people worldwide are suffering from infections acquired in treatment centers, with an estimated 80,000 deaths annually. The actual rates vary from $5 \%$ to $10 \%$ of all patients admitted to modern healthcare centers in the industrialized world up to $25 \%$ in developing countries. The risk of health care-associated infection in developing countries is 2 to 20 times higher than in developed countries [1]

Intensive care units (ICUs) worldwide are encountering the highest density of nosocomial infections (NI) and the spread of antibiotic-resistant pathogens responsible for emerging infection problems in the hospital. In the rank order of pathogens causing ICU-related infections Pseudomonas aeruginosa has held a nearly unchanged position over recent decades. An European survey showed that $P$. aeruginosa is one of the most frequent pathogens isolated from ICU-acquired infections. Several patient and pathogen-specific risk factors are associated with acquisition of this 
pathogen in ICUs, such as length of stay, severity of underlying disease and exposure to invasive procedures, on the other hand, and virulence, adherence, and antimicrobial drug resistance on the other [3].

Prevention of nosocomial infections requires an integrated, monitored, programmed, which includes the following key components: Limiting transmission of organisms between patients in direct patient care through adequate hand washing and glove use, and appropriate aseptic practice, isolation strategies, sterilization and disinfection practices, and laundry, controlling environmental risks for infection, protecting patients with appropriate use of prophylactic antimicrobials, nutrition, and vaccinations, limiting the risk of endogenous infections by minimizing invasive procedures and promoting optimal antimicrobial use, surveillance of infections, identifying and controlling outbreaks, Prevention of infection in staff members, Enhancing staff patient care practices, and continuing staff education. infection control is the responsibility of all healthcare professionals. doctors, nurses, therapists, pharmacists, engineers and others [3].

Methicillin-resistant S. aureus (MRSA) strains have acquired a gene that makes them resistant to nearly all beta-lactam antibiotics. Resistance to other antibiotics is also common, especially in hospital-associated MRSA [4].

Vancomycin resistance in Enterococcus species (VRE) is becoming a major concern in clinical settings as the rate of occurrence of VRE implicated in disease increases. In 1999 the incidence of VRE-mediated nosocomial infections in intensive care units had increased $43 \%$ from that of the period of 1994 to 1998 . Enterococcus faecalis and E. faecium are reported more frequently as etiological agents of disease than are other enterococci, but other species, such as E. avium, occasionally cause disease [5].

MRSA and VRE are amongst the most important bacteria which are resistant to treatment. These bacteria are considered one of the most crucial factors causing nosocomial infections. Infections occur 48 to 72 hours after the patient has been admitted to the hospital provided the patient does not have obvious infection symptoms at the time of admission and the disease is not in its incubation period [6].

Standard interventions to prevent the transmission of MRSA and VRE in health care facilities include hand hygiene, the use of barrier precautions (gloves and gowns) in the care of colonized and infected patients, the use of dedicated instruments and equipment for these patients, and the placement of colonized or infected patients in single rooms or areas reserved for such patients [7].

A number of factors related to infection with resistant microorganisms have been reported, including previous use of antibiotics, corticosteroid therapy, mechanical ventilation, length of hospital stays and use of invasive devices such as catheters. Many researches have indicated that ICU sections are the center of these infections and the number of the patients suffering from nosocomial infection is in fact much greater in ICUs than in other wards. The level of the resistant bacteria has also increased in the ICUs due to repeated use of antibiotics. Nosocomial infections caused by resistant bacteria affect the increase of mortality and also the increase of hospital expenses. Therefore, preventing these bacteria from appearing and spreading is of vital importance for controlling nosocomial infections. Proper supervision, which includes selecting the proper antibacterial, the dose, and also the treatment duration, can prevent or delay the appearance of the antibiotic- resistant bacteria [6].

\section{Extended-spectrum $\beta$-lactamases (ESBLs)}

Are Gram-negative bacteria that produce an enzyme; beta-lactamase that has the ability to break down commonly used antibiotics, such as penicillin's and cephalosporins and render them ineffective for treatment. The ESBLs are broadly classified into three groups: TEM (Temorina Escherichia coli mutant), SHV (Sulfhydr yl variant), and CTX-M (Cefotaximase-munich) types. Klebsiella pneumoniae and Escherichia coli are the major ESBL-producing organisms around the globe [8]. ESBLs are primarily produced by the Enterobacteriaceae family of Gram-negative microbes, in particular Klebsiella pneumonia and Escherichia coli. They are also produced by non-fermentative Gram-negative organisms, such as Acinetobacter baumannii and Pseudomonas aeroginosa and E. coli [9].

\section{Acinetobacter species}

Are gram negative bacteria that belong to the family Moraxellaceae. They generally form part of the normal body flora but can cause a wide variety of local and systemic infections like pneumonia, septicemia and wound infections. The species can often be isolated from reusable medical equipment's such as ventilator tubing, artificial pressure monitoring devices, humidifiers and wash basins. Gram-negative bacteria have the ability to acquire drug resistance genes such as ESBLs, Amp C- $\beta$-lactamase, and carbapenems [10].

\section{Multidrug-resistance (MDR)}

Was defined as acquired non-susceptibility to at least one agent like pseudomonas in three or more antimicrobial categories [11]. Activity against most of aerobic and anaerobic bacteria, including multidrug-resistant (MDR) Gram-negative bacteria. However, Pseudomonas aeruginosa, Proteus spp. and Providencia spp. are intrinsically resistant [12].

Mobile elements are associated with formation of resistance gene clusters in which different determinants that give rise to a multidrug-resistant phenotype are found in close proximity. For example, specific recombination platforms, integrons. can recruit cassettes carrying additional resistance genes via sites specific recombination [13]. 
The prevalence of MDR bacterial species has increased considerably since the introduction followed by arbitrary use of new generation extended spectrum antibiotics like third and fourth generation cephalosporins, carbapenems, monobactams, broad and extended spectrum penicillin's and other antibiotics. Multidrug resistance bacterial isolates have been frequently reported from different parts of the world as an emergence of treatment problem. The World Health Organization (WHO), the European Commission (EU), and the U.S. Centers for Disease Control and Prevention (CDC) have recognized the importance of studying the emergence and determinants of multidrug resistance as well as the need for control [14].

\section{Carbapenem-resistant enterobacteriaceae (CRE)}

Are beta-lactam antimicrobial agents with an exceptionally broad spectrum of activity. Older carbapenems, such as imipenem, were often susceptible to degradation by the enzyme dehydropeptidase-1 (DHP-1) located in renal tubules and required co-administration with a DHP-1 inhibitor such as cystatin. Later additions to the class such as meropenem, ertapenem and Door Opener demonstrated increased stability to DHP-1 and are administered without a DHP-1 inhibitor. Like all beta-lactam antimicrobial agents, carbapenems act by inhibiting bacterial cell wall synthesis by binding to and inactivating penicillin-binding proteins (PBPs) [15].

Community and healthcare associated infections caused by multi-drug resistant gram negative organisms (MDR GN) represent a worldwide threat. Nucleic Acid Detection tests are becoming more common for their detection; however, they can be expensive requiring specialized equipment and local expertise. In study of Chavayda shows that with carefully chosen targets for the detection of resistance genes in MDR GN, efficient and rapid detection is possible. The MT PCR was sensitive and specific and is likely to be more accurate than current phenotypic methods [16].

\section{Objective}

1. Determination of hospital acquired organisms.

2. Identify the pathogenic bacteria from the patients in ICU.

3. Determination the antibiotic sensitivity of the organisms.

4. Determination the percentage of multidrug-resistant bacteria (MDR)

\section{Justification}

To reduce the percentage of MDR of hospital acquired infection

\section{Subjects and Methods \\ Subjects}

Retrospective cohort study was conducted on 137 patients from 1101 patients admitted to ICU and CCU who develop bacterial infection (HAIs patients was 15 patients) different samples were collected from King Faisl medical complex, Taif, KSA during the period from May 2018 to October 2018.

\section{Inclusion criteria}

Patients without bacterial infection on admission ICU and CCU department.

\section{Exclusion criteria}

Patients with bacterial infection on admission ICU and CCU department.

\section{Methods}

Culturing the samples on its specific agars:

Types of samples:

- Urine on CLED and blood agar.

- Sputum on chocolate, MacConkey and blood agars.

- Vaginal swab on MacConkey, blood, chocolate, SDA.

- Wound swab on MacConkey and blood agars.

- $\quad$ CSF 'cerebrospinal fluid ' 2blood (aerobic + an aerobic), MacConkey, chocolate agars.

- Body fluids' 2blood (aerobic + an aerobic), MacConkey, chocolate agars. and make slide for gram stain.

- Catheter' 2 blood agar (aerobic + an aerobic), MacConkey, chocolate agars. and make slide for gram stain.

- Tracheal sample on chocolate, MacConkey and blood agars.

- Incubation for 24 hours, then reading the growth If the samples were positive. Making sensitivity test by phonix.

- $\quad$ Blood culture" test."

- 2 bottels

- $\quad$ aerobic*anaerobic.

- Put it in BACTEC instrument and incubate it for 7 days then culture the positive samples According to the bottles (aerobic + an aerobic)

- Aerobic > culture it on blood, MacConkey, chocolate agars.

- Anaerobic > culture it on 2 blood, MacConkey, chocolate agars.

- Then make gram stain from the positive sample bottle.

Statistical analysis

Collected data was be coded, tabulated and introduced to a PC using the Statistical Package for Social Science (SPSS) for windows version 25.

\section{Ethical concerns}

Ethical approval for this work was be obtained from the Ethics Review Committee of the King Faisal Medical Complex and Taif University, Taif, KSA.

\section{Result}

Our study included 137 from 1101 patients in ICU and CCU with bacterial infection. There are 15 patients caught HAIs. Their age ranged from 20 to 85 years, (80\%) male, (20\%) female. 


\section{Discussion}

Hospital-acquired infections (HAI) have been recognized for over a century as a critical problem affecting the quality of healthcare, and they constitute a major source of adverse healthcare outcomes. The emergence of multidrug-resistant bacteria (MDR) has become a public health problem, creating a new burden on medical care in hospitals, particularly for patients admitted to intensive care units (ICU). In critical care units, there is extensive antimicrobial use, which imposes a selection pressure and promotes the emergence of MDR. In addition to this, ICU patients have an increased risk of infection due to their underlying diseases or conditions, impaired immunity, and exposure to multiple invasive devices (mechanical ventilation, central venous catheters (CVC), and urinary tract catheters) [17].

The incidence of ICU-HAI is 5 -10-times higher than HAI rates in general wards. HAI in the ICU has been associated with increased morbidity, mortality, and costs. Antibiotic resistance is when bacteria develop the ability to resist the bactericidal or bacteriostatic effects of one or more antibiotic class (multidrug resistance
(MDR). This resistance is most commonly noted in intensive care units (ICUs), which is due to the widespread use of antibiotics in these units compared to the other hospital departments. A study found that the incidence of ICU nosocomial infections worldwide was between 5\% - 30\%. According to the national healthcare safety network report in the United States (US); age, comorbid diseases, duration of hospitalization, length of ICU stay, immune status, and disease severity are all considered host risk factors for developing nosocomial infections in ICUs [17].

In (Table 1) shows the most frequent isolated (+ve) organisms were $12(9 \%)$ Staph aureus, the $1 \%$ of these were MRSA and in (Table 2) shows the most frequent isolated (-ve) organisms were $31(23 \%)$ Klebsiella Spp, the $12 \%$ of these were ESBL producers, while the study of Juarez., et al. 2015 shows the S. aureus ( $=32$, $12 \%$; $90.6 \%$ of these were MRSA) and the E. coli (n $=54,20 \%$; $94.4 \%$ of these were ESBL producers). the difference between our study and Juarez., et al. 2015 study was search duration. The period of our study was 6 months but in Juarez., et al. [17] study, the period was 5 years.

\begin{tabular}{|c|c|c|c|c|c|}
\hline Organism & Staph aureus & Staph epidermidis) & $\begin{array}{l}\text { Staph aureus } \\
\text { (MSSA) }\end{array}$ & $\begin{array}{c}\text { Enterococcus } \\
\text { faecium }\end{array}$ & $\begin{array}{c}\text { Streptococcus } \\
\text { pneumonia }\end{array}$ \\
\hline Number Tested & $12(8.7 \%)$ & $6(4.3 \%)$ & $1(0.7 \%)$ & $3(2.1 \%)$ & $1(0.7 \%)$ \\
\hline \multicolumn{6}{|l|}{ Susceptibility \% } \\
\hline Gentamycin & $10(83 \%)$ & $2(33 \%)$ & $1(100 \%)$ & --- & --- \\
\hline Imipenem & $8(67 \%)$ & $1(17 \%)$ & --- & --- & --- \\
\hline Cefoxitin & $1(8 \%)$ & $1(17 \%)$ & --- & --- & --- \\
\hline Cefotaxime & $8(67 \%)$ & $1(17 \%)$ & --- & --- & --- \\
\hline Ampicillin & --- & --- & --- & --- & --- \\
\hline Penicillin & --- & --- & --- & --- & --- \\
\hline Oxacillin & $8(67 \%)$ & $1(17 \%)$ & --- & --- & --- \\
\hline Augmentin & $4(33 \%)$ & $1(17 \%)$ & --- & --- & --- \\
\hline Daptomycin & $12(100 \%)$ & $6(100 \%)$ & $1(100 \%)$ & $3(100 \%)$ & --- \\
\hline $\begin{array}{l}\text { Trimethoprim/ } \\
\text { sulfamesoxazole }\end{array}$ & $12(100 \%)$ & $4(67 \%)$ & $1(100 \%)$ & --- & --- \\
\hline Teicoplanin & $12(100 \%)$ & $4(67 \%)$ & $1(100 \%)$ & $3(100 \%)$ & --- \\
\hline Vancomycin & $12(100 \%)$ & $6(100 \%)$ & $1(100 \%)$ & $3(100 \%)$ & --- \\
\hline Clindamycin & $12(100 \%)$ & $4(67 \%)$ & $1(100 \%)$ & --- & --- \\
\hline Erythromycin & $10(83 \%)$ & $1(17 \%)$ & $1(100 \%)$ & --- & --- \\
\hline Linzolid & $12(100 \%)$ & $6(100 \%)$ & $1(100 \%)$ & $3(100 \%)$ & --- \\
\hline Ciprofloxacin & $6(50 \%)$ & $3(50 \%)$ & $1(100 \%)$ & --- & --- \\
\hline Moxifloxacin & $10(83 \%)$ & $4(67 \%)$ & $1(100 \%)$ & --- & --- \\
\hline Rifampicin & $12(100 \%)$ & $5(83 \%)$ & $1(100 \%)$ & --- & --- \\
\hline Tetracycline & $12(100 \%)$ & $5(83 \%)$ & $1(100 \%)$ & $3(100 \%)$ & --- \\
\hline Nitrofurantoin & $11(92 \%)$ & $5(83 \%)$ & $1(100 \%)$ & --- & --- \\
\hline Meropenem & $1(8 \%)$ & $1(17 \%)$ & --- & --- & --- \\
\hline Cefepime & $1(8 \%)$ & $1(17 \%)$ & --- & --- & --- \\
\hline Mupirocin high level & $4(33 \%)$ & $3(50 \%)$ & $1(100 \%)$ & --- & --- \\
\hline Amoxicillin-Clavulanate & $4(33 \%)$ & --- & --- & --- & --- \\
\hline
\end{tabular}

Table 1: Frequency of susceptibility of isolated Gram +ve microorganisms to different classes of antibiotic. 


\begin{tabular}{|c|c|c|c|c|c|c|c|c|c|c|c|c|}
\hline & $\begin{array}{l}\frac{2}{2} \\
\text { के } \\
\frac{\pi}{0} \\
\frac{0}{5} \\
\frac{0}{0} \\
\frac{0}{2}\end{array}$ & 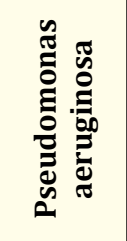 & 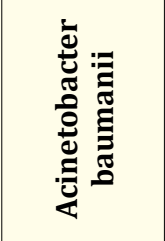 & 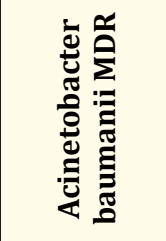 & 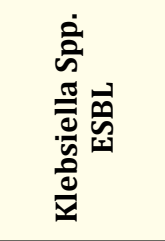 & 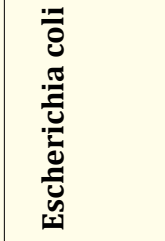 & 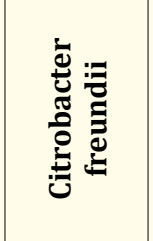 & 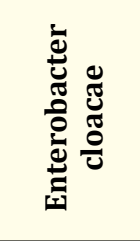 & 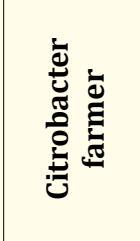 & 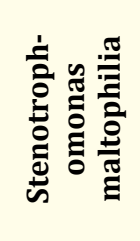 & 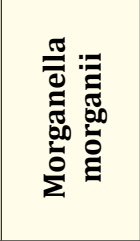 & 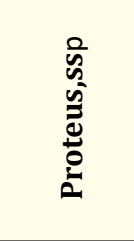 \\
\hline Number tested & $31(22.6 \%)$ & $11(8 \%)$ & $14(10.2 \%)$ & $19(13.8 \%)$ & $16(11.6 \%)$ & $14(10.2 \%)$ & $1(0.7 \%)$ & $2(1.5 \%)$ & $1(0.7 \%)$ & $2(1.5 \%)$ & $1(0.7 \%)$ & $1(0.7 \%)$ \\
\hline Amikacin & $10(32 \%)$ & $7(63 \%)$ & $1(7.1 \%)$ & $1(5 \%)$ & $7(43 \%)$ & $6(42 \%)$ & $1(100 \%)$ & $2(100 \%)$ & $1(100 \%)$ & --- & $1(100 \%)$ & $1(100 \%)$ \\
\hline Gentamycin & $9(29 \%)$ & $7(63 \%)$ & --- & $1(5 \%)$ & $7(43 \%)$ & $5(36 \%)$ & $1(100 \%)$ & $2(100 \%)$ & $1(100 \%)$ & --- & $1(100 \%)$ & $1(100 \%)$ \\
\hline Ertapenem & $7(23 \%)$ & --- & --- & --- & $5(31 \%)$ & $6(42 \%)$ & \begin{tabular}{|l|}
--- \\
\end{tabular} & $2(100 \%)$ & $1(100 \%)$ & --- & $1(100 \%)$ & $1(100 \%)$ \\
\hline Imipenem & $6(19 \%)$ & $6(54 \%)$ & --- & $1(5 \%)$ & $5(31 \%)$ & $6(42 \%)$ & --- & $2(100 \%)$ & --- & --- & --- & $1(100 \%)$ \\
\hline Meropenem & $6(19 \%)$ & $6(54 \%)$ & --- & $1(5 \%)$ & $5(31 \%)$ & $6(42 \%)$ & --- & $2(100 \%)$ & --- & --- & --- & --- \\
\hline Cephalothin & $2(6 \%)$ & --- & --- & --- & $1(6 \%)$ & --- & --- & --- & --- & --- & --- & --- \\
\hline Cefuroxime & $3(10 \%)$ & --- & --- & --- & $1(6 \%)$ & $2(14 \%)$ & --- & --- & --- & --- & --- & --- \\
\hline Cefoxitin & $9(29 \%)$ & --- & --- & --- & $7(37 \%)$ & $3(21 \%)$ & --- & --- & --- & --- & $1(100 \%)$ & $1(100 \%)$ \\
\hline Ceftazidime & $6(19 \%)$ & $4(36 \%)$ & --- & $1(5 \%)$ & $8(50 \%)$ & $4(29 \%($ & --- & $2(100 \%)$ & --- & $1(50 \%)$ & $1(100 \%)$ & $1(100 \%)$ \\
\hline Ceftriaxone & $5(16 \%)$ & --- & --- & $1(5 \%)$ & $2(12 \%)$ & $2(14 \%)$ & --- & $2(100 \%)$ & --- & --- & $1(100 \%)$ & --- \\
\hline Cefepime & $5(16 \%)$ & $4(36 \%)$ & --- & $1(5 \%)$ & $2(12 \%)$ & $3(21 \%)$ & $1(100 \%)$ & $2(100 \%)$ & --- & --- & $1(100 \%)$ & $1(100 \%)$ \\
\hline Aztreonam & $6(19 \%)$ & $3(27 \%)$ & --- & --- & $3(19 \%)$ & $3(21 \%)$ & --- & $2(100 \%)$ & --- & --- & $1(100 \%)$ & $1(100 \%)$ \\
\hline Ampicillin & --- & --- & --- & --- & $2(12 \%)$ & --- & --- & --- & --- & --- & --- & --- \\
\hline Augmentin & $4(13 \%)$ & --- & --- & --- & $4(25 \%)$ & $1(7 \%)$ & --- & --- & --- & --- & --- & $1(100 \%)$ \\
\hline $\begin{array}{l}\text { Piperacillin- } \\
\text { tazobactam }\end{array}$ & $16(9 \%)$ & $3(27 \%)$ & --- & $1(5 \%)$ & $5(31 \%)$ & $5(35 \%)$ & --- & $2(100 \%)$ & --- & --- & $1(100 \%)$ & $1(100 \%)$ \\
\hline Colistin & $1(3 \%)$ & $8(72 \%)$ & $9(64.2 \%)$ & $5(26 \%)$ & $1(6 \%)$ & --- & --- & $1(50 \%)$ & --- & --- & --- & --- \\
\hline $\begin{array}{l}\text { Trimethoprim/ } \\
\text { Sulfamesoxa- } \\
\text { zole }\end{array}$ & $6(19 \%)$ & --- & --- & $1(5 \%)$ & $3(19 \%)$ & --- & $1(100 \%)$ & $1(50 \%)$ & --- & $2(100 \%)$ & --- & --- \\
\hline Nitrofurantoin & $5(16 \%)$ & --- & --- & --- & $4(25 \%)$ & $5(35 \%)$ & $1(100 \%)$ & --- & $1(100 \%)$ & --- & --- & --- \\
\hline Ciprofloxacin & $9(29 \%)$ & $6(54 \%)$ & --- & $1(5 \%)$ & $4(25 \%)$ & $4(28 \%)$ & --- & $1(50 \%)$ & --- & --- & --- & --- \\
\hline Levofloxacin & $10(32 \%)$ & $6(54 \%)$ & --- & $1(5 \%)$ & $5(31 \%)$ & $4(28 \%)$ & $1(100 \%)$ & $1(50 \%)$ & --- & $2(100 \%)$ & --- & --- \\
\hline Tigecyclin & $10(32 \%)$ & --- & --- & --- & $10(62 \%)$ & $6(43 \%)$ & $1(100 \%)$ & $2(100 \%)$ & $1(100 \%)$ & --- & --- & --- \\
\hline
\end{tabular}

Table 2: Frequency of susceptibility of isolated Gram -ve microorganisms to different classes of antibiotic.

While in study of Thuy., et al. [18] shows the most frequent isolated is $1,550(40.4 \%)$ were $E$. coli, the $39.8 \%$ of these were ESBL producers. Compared with our search the E. coli was $14(10.2 \%), 3.6 \%$ of these were ESBL producers, they may be also due to period of study.
The most frequent sample were taken is sputum and isolated the largest proportion of the K. pneumonia as in (Table 3). Compared with our search the study of Thuy., et al. 2018 shows the most frequent sample is swabs (123 nasal swabs, 199 rectal swabs). they may be due to the variation number of the sample and period of study.

\begin{tabular}{|c|c|c|c|c|c|c|c|c|c|c|c|c|c|c|c|}
\hline 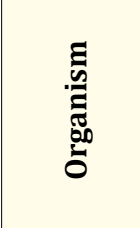 & $\begin{array}{l}0 \\
\vdots \\
\vdots \\
\vdots \\
\tilde{J} \\
\dot{\omega}\end{array}$ & 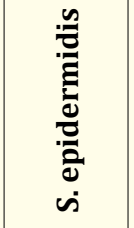 & 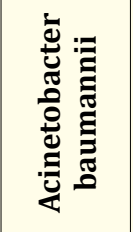 & 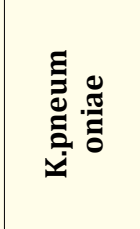 & & 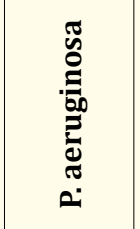 & 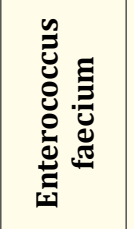 & 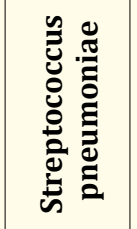 & $\underset{\overline{8}}{:}$ & 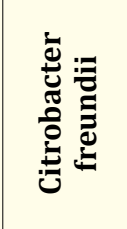 & 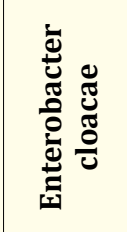 & 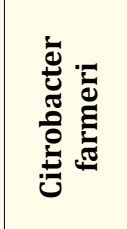 & 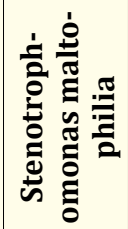 & 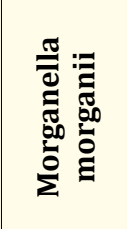 & 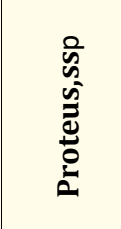 \\
\hline Sputum & $5(3.6 \%)$ & --- & $11(8 \%)$ & $23(17 \%)$ & $2(1.5 \%)$ & $9(6.5 \%$ & --- & $2(1.5 \%)$ & --- & --- & --- & $1(0.7 \%)$ & $1(0.7 \%)$ & --- & --- \\
\hline Urine & --- & --- & --- & $10(7 \%)$ & --- & $1(0.7 \%)$ & $3(2.2 \%)$ & --- & $9(6.5 \%)$ & --- & --- & --- & \begin{tabular}{|c|}
-- \\
\end{tabular} & --- & --- \\
\hline $\begin{array}{l}\text { Blood } \\
\text { culture }\end{array}$ & $3(2 \%)$ & $5(3.6 \%)$ & $6(4.5 \%)$ & $6(4.5 \%)$ & --- & --- & --- & --- & $3(2.2 \%)$ & --- & --- & --- & $1(0.7 \%)$ & --- & --- \\
\hline Wound & $5(3.6 \%)$ & $1(0.7 \%)$ & $6(4.5 \%)$ & $2(1.5 \%)$ & --- & $1(0.7 \%)$ & --- & --- & $2(1.5 \%)$ & $1(0.7 \%)$ & $1(0.7 \%)$ & --- & --- & $1(0.7 \%)$ & $1(0.7 \%)$ \\
\hline $\begin{array}{l}\text { Body } \\
\text { fluid }\end{array}$ & --- & --- & --- & $2(1.5 \%)$ & --- & --- & --- & --- & --- & --- & $1(0.7 \%)$ & --- & --- & --- & --- \\
\hline Tracheal & --- & --- & $3(2.2 \%)$ & --- & --- & --- & --- & --- & --- & --- & --- & --- & & --- & --- \\
\hline Catheter & --- & --- & $4(2.9 \%)$ & --- & $1(0.7 \%)$ & --- & --- & --- & --- & --- & --- & --- & --- & --- & --- \\
\hline CSF & --- & --- & \begin{tabular}{|l|}
--- \\
\end{tabular} & $1(0.7 \%)$ & \begin{tabular}{|c|}
-- \\
\end{tabular} & --- & --- & --- & --- & --- & --- & --- & --- & --- & --- \\
\hline Swap & --- & --- & --- & $3(2.2 \%)$ & --- & --- & --- & --- & --- & --- & --- & --- & --- & --- & --- \\
\hline
\end{tabular}

Table 3: Frequency of isolated microorganisms in different biological samples. 
In (Table 4) shows the Frequency of resistant isolated microorganisms to MDR is Acinetobacter baumannii 14(10.2\%), resistant to MRSA is Staph aureus $1(0.7 \%)$, resistant to VRE are Staph epidermidis $1(0.7 \%)$ and Enterococcus faecium $1(0.7 \%)$, resistant to ESBL are Klebsiella pneumoniae 40(29\%), Escherichia coli 5(4\%), Citrobacter farmeri $1(0.7 \%)$ and Klebsilla, oxytoca $1(0.7 \%)$. In study of Juarez., et al. [17] the microorganism's resistant to MDR are P. aeruginosa $14 \%$ and Acinetobacter baumannii, $6 \%$ resistant to ESBL is E. coli $94.4 \%$, resistant to MRSA is $S$. aureus $90.6 \%$, resistant to VRE is E. faecium 18.7\%. while in study of Thuy., et al. [18] shows the microorganisms resistant to ESBL is E. coli was $39.8 \%$, resistant to carbapenemase is Acinetobacter spp. $22.0 \%$. These percentages vary due to the risk of infection with MDRB has been related to a number of factors, including previous antimicrobial therapy, cross-transmission, and length of hospital stay.
The Frequency of HAIs microorganisms during 6 months in Taif was 15 (10.9\%) in (Table 5). Compared with our search the study of Iliyasu., et al. 2018 shows the percentage of HAIsin Nigeria was 518(6.3\%.) There was a high burden of HAI especially UTI in this hospital with resistance to commonly used antibiotics. In developed countries, HAI rates of $5 \%$ to $15 \%$, sometimes up to $50 \%$, have been reported among hospitalized patients in the regular wards and intensive care units (ICUs) respectively. In developing countries, the problem is likely much higher, and yet, the magnitude of the problem remains underestimated or even unknown largely because HAI diagnosis is complex and surveillance activities which requires expertise and resources, are lacking in most of these countries. Furthermore, infection control practices remain rudimentary as most hospitals lack effective infection control programs and trained professionals [19].

\begin{tabular}{|l|c|c|c|c|c|c|c|c|c|}
\hline Organism & $\begin{array}{c}\text { Staph } \\
\text { aureus }\end{array}$ & $\begin{array}{c}\text { Staph } \\
\text { epidermidis }\end{array}$ & $\begin{array}{c}\text { Acinetobacter } \\
\text { baumannii }\end{array}$ & $\begin{array}{c}\text { Klebsiella } \\
\text { pneumoniae }\end{array}$ & $\begin{array}{c}\text { Enterococcus } \\
\text { faecium }\end{array}$ & $\begin{array}{c}\text { Escherichia } \\
\text { coli }\end{array}$ & $\begin{array}{c}\text { Citrobacter } \\
\text { freundii }\end{array}$ & $\begin{array}{c}\text { Citrobacter } \\
\text { farmeri }\end{array}$ & $\begin{array}{c}\text { Klebsilla, } \\
\text { oxytoca }\end{array}$ \\
\hline$M D R$ & --- & --- & $14(10.2 \%)$ & --- & --- & --- & --- & --- & --- \\
\hline$M R S A$ & $1(0.7 \%)$ & --- & --- & --- & --- & --- & --- & --- & --- \\
\hline$V R E$ & ---- & $1(0.7 \%)$ & --- & --- & $1(0.7 \%)$ & --- & --- & --- & --- \\
\hline$E S B L$ & ---- & --- & --- & $40(29.2 \%)$ & --- & $5(3.6 \%)$ & --- & $1(0.7 \%)$ & $1(0.7 \%)$ \\
\hline$C R E$ & --- & --- & --- & $1(0.7 \%)$ & --- & --- & $1(0.7 \%)$ & --- & --- \\
\hline$M R S$ & --- & $1(0.7 \%)$ & --- & --- & --- & --- & -- & -- & --- \\
\hline
\end{tabular}

Table 4: Frequency of resistant isolated microorganisms to different classes of antibiotic.

\begin{tabular}{|c|c|c|c|c|c|c|}
\hline Months & May & June & July & August & September & October \\
\hline Percentage of HAIS & $2(13.3 \%)$ & $1(6.7 \%)$ & $2(13.3 \%)$ & $6(40 \%)$ & $4(26.7 \%)$ & $0(0 \%)$ \\
\hline
\end{tabular}

Table 5: Frequency of isolated HAIS microorganisms during the study period.

While the study of Thuy., et al. [18] the proportion of ICU patients with HAIs was 85 (23.4\%). the difference of result was due to the shortness of period and Varity of geographical area

\section{Conclusion}

The emergence of MDR bacteria poses a difficult task for physicians, who have limited therapeutic options. And therefore a continuous surveillance program to observe the emergence of different bacterial resistance patterns is advised to establish unified guidelines across Saudi Arabia to reduce further progress in the emergence of MDR organizations.

\section{Bibliography}

1. Nazir A and Kadri S. "An overview of hospital acquired infections and the role of the microbiology laboratory". International Journal of Research in Medical Sciences 2.1 (2014): 21.
2. Plowman R., et al. "The rate and cost of hospital-acquired infections occurring in patients admitted to selected specialties of a district general hospital in England and the national burden imposed". Journal of Hospital Infection 47.3 (2001): 198-209.

3. Agodi A., et al. "Pseudomonas aeruginosa carriage, colonization, and infection in ICU patients". Intensive Care Medicine 33.7 (2007): 1155-1161.

4. Acton D., et al. "Intestinal carriage of Staphylococcus aureus: how does its frequency compare with that of nasal carriage and what is its clinical impact?" European Journal of Clinical Microbiology and Infectious Diseases 28.2 (2009): 155-127.

5. Young S., et al. "Vancomycin-Resistant Enterococci and Bacterial Community Structure following a Sewage Spill into an Aquatic Environment". Applied and Environmental Microbiology 82.18 (2016): 5653-5660. 
6. Hosseini JM., et al. "Antibiotic Resistance Pattern of Bacteria Isolated from Nosocomial Infection in Internal, Surgery and Neurosurgery Intensive Care Unit (NICU) at a Tertiary Care Hospital in Tehran, Iran". Biosciences Biotechnology Research Asia 41.3 (2017): 1095-1102.

7. Huskins W. "Interventions to prevent transmission of antimicrobial-resistant bacteria in the intensive care unit". Current Opinion in Critical Care, 13.5 (2007): 572-577.

8. Rottier W., et al. "Effects of confounders and intermediates on the association of bacteraemia caused by extended-spectrum $\beta$-lactamase-producing Enterobacteriaceae and patient outcome: a meta-analysis". Journal of Antimicrobial Chemotherapy 67.6 (2012):1311-1320.

9. Cagnacci S., et al. "European Emergence of CiprofloxacinResistant Escherichia coli Clonal Groups 025:H4-ST 131 and 015:K52:H1 Causing Community-Acquired Uncomplicated Cystitis". Journal of Clinical Microbiology 46.8 (2008): 26052612.

10. Ghanem S., et al. "Antimicrobial resistance pattern of Acinetobacter spp. isolated from clinical samples in a tertiary care hospital at Madinah, Saudi Arabia". African Journal of Microbiology Research 12.30 (2018): 723-729.

11. De Pascale G., et al. "High dose tigecycline in critically ill patients with severe infections due to multidrug-resistant bacteria". Critical Care 10.18 (2014): 1-9.

12. Cai B., et al. "Prevalence of Carbapenem-Resistant Gram-Negative Infections in the United States Predominated by Acinetobacter baumannii and Pseudomonas aeruginosa". Open Forum Infectious Diseases 4.3 (2017).

13. He S., et al. "Insertion Sequence IS26 Reorganizes Plasmids in Clinically Isolated Multidrug-Resistant Bacteria by Replicative Transposition". MBIO 6.3 (2018): 1-14.

14. Sah B., et al. "Drug Resistance Pattern of Bacterial Pathogens of Enterobacteriaceae Family". Tribhuvan University Journal of Microbiology 4 (2018): 15-22.

15. Zhanel G., et al. "Comparative Review of the Carbapenems". Drugs 67.7 (2007): 1027-1052.

16. Chavada R and Maley M. "Evaluation of a Commercial Multiplex PCR for Rapid Detection of Multi Drug Resistant GramNegative Infections". The Open Microbiology Journal 9.1 (2015): 125-135.

17. Juáreza PC., et al. "The impact of hospital-acquired infections with multidrug-resistant bacteria in an oncology intensive care unit". International Journal of Infectious Diseases 31 (2015): 31-34.
18. Thuy D., et al. "Hospital-acquired colonization and infections in a Vietnamese intensive care unit". Plos One 13.9 (2018): e0203600.

19. Iliyasu G., et al. "Laboratory-confirmed hospital-acquired infections: An analysis of a hospital's surveillance data in Nigeria". Heliyon 4.8 (2018): e00720.

\section{Volume 2 Issue 12 December 2019 (C) All rights are reserved by Khadiga Ahmed Ismail.,} et al. 